\title{
How Speaking Creates Droplets That May Spread COVID-19
}

\author{
High-speed video reveals the process that produces airborne saliva \\ droplets during speech and also shows that lip balm can reduce droplet \\ production.
}

By David Ehrenstein

$\mathrm{O}$ ne way that COVID-19 spreads is through saliva droplets emitted during speech, but the precise mechanism that creates the droplets in the mouth is not well understood. Now Manouk Abkarian of the University of Montpellier, France, and Howard Stone of Princeton University have recorded high-speed video of the mouth of a volunteer speaking various sounds [1]. The duo uncovered the droplet production process and identified the consonants, such as " $p$ " and "t," that most effectively generate droplets when spoken. They also found that lip balm interferes with droplet formation and can dramatically reduce the number of droplets emitted.

Abkarian and Stone found that when the mouth makes the sounds "p" or "b," saliva forms a thin sheet across the lip gap, and this sheet quickly breaks up into vertical filaments. The researchers determined that a property of saliva called

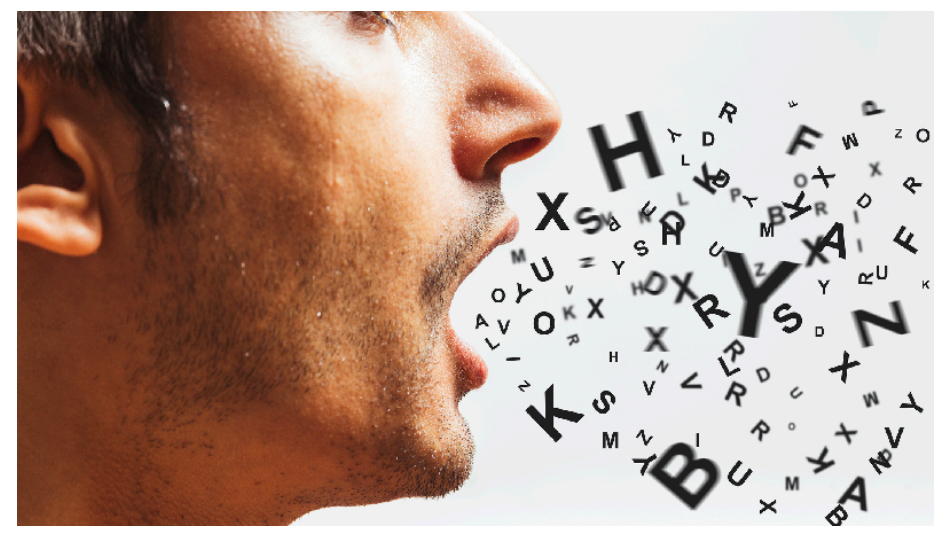

Pronouncing the sound "Pa" causes a film of saliva to form across the gap between the lips, but it quickly breaks apart into a series of vertical filaments that are then stretched and eventually dislodged by airflow. Surface tension later causes each flying filament to break up into a line of droplets (not shown).

Credit: M. Abkarian and H. A. Stone [1]

viscoelasticity is critical in that it allows these filaments to survive and stretch out as they experience air blowing through the lips, even though they eventually become dislodged. The stretched, airborne filaments then fall apart into lines of droplets, thanks to surface tension. These two sounds, along with "t" and "d," produce the most droplets because they involve a burst of air through a narrow saliva-filled space-either between the lips or between the tongue and palate. In contrast, the sound "m," as in "mama," sends air The worst you could say. Certain consonants, such as "p" and "b," produce more droplets than others, according to experiments. Credit: jaouad.K/Getty Images mainly through the nose, so it produces very few droplets, according to the team's results.

After analyzing the physical properties of saliva and its 


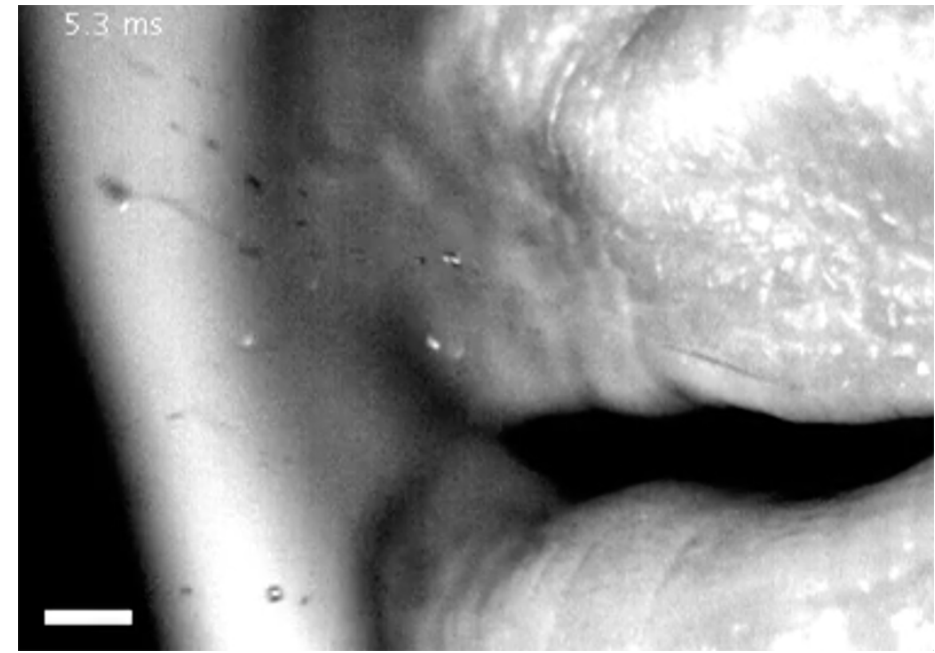

This angle allows a clear view of the droplets formed from pronouncing the sound "Pa."

Credit: M. Abkarian and H. A. Stone [1]

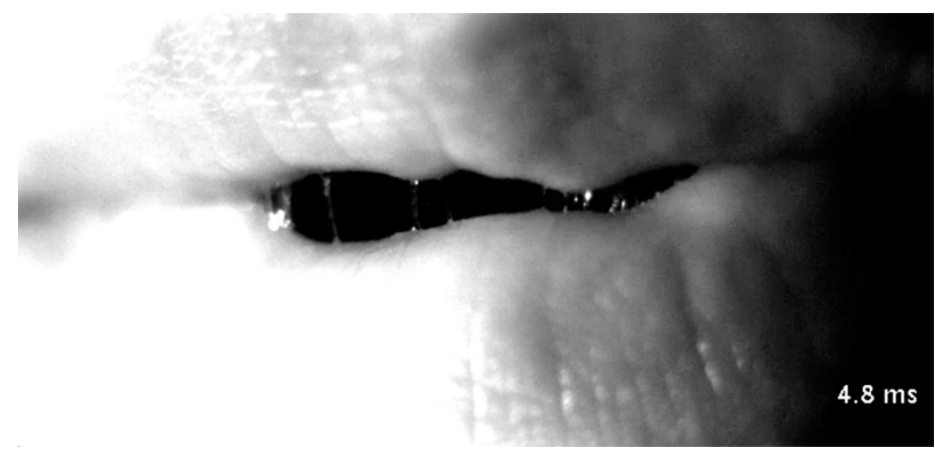

Very few droplets are expelled by pronouncing the sound "Ma" because the airflow for the nasal consonant " $\mathrm{m}$ " is directed mainly through the nose.

Credit: M. Abkarian and H. A. Stone [1] interactions with the lips, the pair showed that lip balm destabilizes filament formation on the lips and, at least for one individual, reduces by four times the number of sprayed droplets. They suggest that a balm could be developed as a cheap and effective mitigation strategy. On the other hand, Stone and Abkarian speculate that COVID-19 superspreaders might be individuals whose saliva has a viscoelasticity that optimizes the number of droplets expelled during speech. Taking this thinking even further, Stone wonders if evolution could have taken advantage of the critical role of salivary properties. "We are speculating whether the virus is also causing some rheological modifications of saliva that may be enhancing the eventual transport of the virus," he says.

David Ehrenstein is the Focus Editor for Physics.

\section{REFERENCES}

1. M. Abkarian and H. A. Stone, "Stretching and break-up of saliva filaments during speech: A route for pathogen aerosolization and its potential mitigation," Phys. Rev. Fluids 5, 102301 (2020). 